\title{
Portada / Revisores / Índice
}

FAVE Sección Ciencias Agrarias es la revista oficial de la Facultad de Ciencias Agrarias de Esperanza (Universidad Nacional del Litoral) y tiene por objetivo publicar trabajos originales e inéditos, pudiendo ser artículos científicos, comunicaciones, revisiones y notas, brindando un medio que permita a los investigadores nacionales e internacionales publicar sus resultados, contribuyendo al desarrollo científico-tecnológico en el campo disciplinar de las Ciencias Agrarias y Forestales.

La revista es de frecuencia semestral, publicándose un volumen por año con dos números (Enero-Junio y Julio-Diciembre). El idioma oficial de la revista es el español, aceptándose además trabajos

en portugués e inglés

FAVE - Sección Ciencias Agrarias es continuación de la Revista FAVE (ISSN 0325 3112), Publicación que fuera incorporada al Núcleo Básico de Revistas Científicas Argentinas del Consejo Nacional de Investigaciones Científicas y Técnicas (CONICET), Scielo, DOAJ, REDIB, LATINDEX catálogo 2.0 y DIALNET.

La Revista FAVE - Sección Ciencias Agrarias agradece a los siguientes revisores el arbitraje de los artículos evaluados en este período:

Dra. María del Valle Basanta. AER Esperanza, EEA Rafaela, CR Santa Fe, Argentina Lic. Natalia Cáceres. Laboratorio de Recursos Genéticos y Sustentabilidad Bioclimática; IRNASUS, Universidad Católica de Córdoba-CONICET, Argentina

Ph.D. Rodrigo Orlando Campo-Arana. Facultad de Ciencias Agrícolas (UNICORDOBA), Montería, Córdoba, Colombia

MSc. María Florencia Casse. INTA Colón e INTA EEA Concordia, Entre Ríos, Argentina Ph.D. Jairo Castaño-Zapata. Profesor Distinguido, Universidad de Caldas, Colombia Dr. Eduardo de Sá Pereira. INTA AER Coronel Suárez, EEA Cesáreo Naredo, Coronel Suárez, Buenos Aires, Argentina

Dr. Marcelino de Souza. Facultad de Ciencias Económicas (UFRGS), Brasil Msc. Julio Denoia. Facultad de Ciencias Agrarias (UNR), Argentina Dr. Álvaro Díaz Barrera. Pontificia Universidad Católica de Valparaíso, Chile Dra. Gabriela Rosa Facciuto. Instituto de Floricultura, INTA-UNLZ, Argentina Lic. Laura Beatriz Gastaldi. INTA EEA Rafaela, Santa Fe, Argentina Dra. Ludmila La Manna. Facultad de Ingeniería - UNPSJB CONICET, Argentina Mg. Gabriela Maria Litwin. INTA AER Crespo, EEA Paraná, CRER, Argentina Dra. Marlen Martínez-Domínguez. CIESAS, Oaxaca de Juárez, Oaxaca, México MSc. Federico A. Massoni. INTA EEA Rafaela, Santa Fe, Argentina Dra. Mariel Mitidieri Laboratorio Fitopatología. INTA EEA San Pedro, Argentina MS. Walter Nievas. EEA INTA Alto Valle, Río Negro, Argentina MSc. Natalia Rodriguez Castillo. Universidad Nacional de Colombia Dr. María Dolores Rodriguez-Torres. Instituto Politécnico Nacional, CIIDIR, Unidad Michoacán, México

Dr. Juan Ignacio Romero Cabrera. CENUR LN, Universidad de la República, Uruguay Dra. Adriana Saluso. INTA EEA Paraná, Entre Ríos, Argentina Máster Ricardo Enrique Tamara Morelos. Corporación Colombiana de investigación agropecuaria AGROSAVIA, Colombia Dra. Alicia Florencia Urteaga Omar. Facultad de Ciencias Agropecuarias (UNER), Argentina Dra. María Stella Zerbino. Investigadora jubilada, INIA, Montevideo, Uruguay 


\section{INDICE}

\section{Volumen 21, $\mathrm{N}^{0}$ 1, 2022}

Factores del contexto que afectan la competitividad de empresas lecheras del centro de Santa Fe.

Acetta P M, Castignani MI, Rossler N

Caracterización morfológica y evaluación de hongos poscosecha del ostiolo del higo variedad "Guarinta".

Buttarelli MS, Stegmayer MI, Trod B, Castro D, Bouzo CA

Influencia de un complejo viral en rendimiento y contenido de clorofila de dos variedades de batata.

Buxmann E, Vilanova Perez A, López Colomba E, Suasnabar R, Luque A, Araujo Vieira de Souza JC, Di Feo L

Efectos de cultivos de cobertura sobre el agua del suelo, el aporte de carbono al sistema y el rendimiento de soja en diferentes ambientes.

Capurro JE, Montico S

Optimización de medios de cultivos con fuentes agroindustriales para el crecimiento del ñame espino (Dioscorea rotundata Poir)

Luna Castellanos LL, Sánchez López DB

Obtención de híbridos de Portulaca umbraticola a través de cruzamiento convencional Sandí Montero H, Zúñiga Orozco A, Carrodeguas Gonzalez A

Uso de suelo en laderas del sector sur de la Comuna de Lonquimay (Chile) y sus características estructurales

Vial-Alarcón M 99 